\section{More than 70 ways to show resilience}

Ruben Dahm and his colleagues call for greater "flood resilience" in delta cities (Nature 516, 329; 2014). But achieving resilience depends on what we mean by it: there are more than 70 definitions in the scientific literature.

These definitions vary between two extremes, with most trying to achieve a balance between the two (M. de Bruijne et al. in Designing Resilience 13-32; Univ. Pittsburgh Press, 2010). Dahm et al.

implicitly define resilience as the ability of a system to bounce back after stress, as do many politicians and the World Economic Forum in Geneva, Switzerland.

At the other extreme, resilience is seen as "the capacity of social-ecological systems to adapt or transform in response to unfamiliar, unexpected and extreme shocks" as proposed by ecologist Stephen Carpenter and economics Nobel laureate Kenneth Arrow, among others (S. R. Carpenter et al. Sustainability 4, 3248-3259; 2012).

To recover, to adapt or to invest in both possibilities? All make sense in the right context. We might want a city to recover from flooding, for example, or the world to adapt to the effects of climate change.

Long-term policies to promote either recovery or adaptation, or prepare for both, are likely to be very different. We must have a clear definition from the outset. Len Fisher University of Bristol, UK. len.fisher@bristol.ac.uk

\section{Labs leak staff under French law}

Ironically, a well-intentioned law enacted in France almost three years ago to protect the poorly qualified is preventing young researchers from completing their postdoctoral training. In my view, research institutions should be exempted from its requirements.

Called the Sauvadet law, it stipulates that a worker must be appointed to a permanent position after six years of shortterm contracts in the public sector. Since the law came into effect in March 2012 (and retrospectively), publicly funded research institutions - including INSERM and the CNRS - have limited the number of postdocs becoming eligible for tenure by not renewing contracts if a postdoc has already worked there for three years (see go.nature. com/vki6fq and go.nature.com/ navteh; both in French).

Young researchers are therefore being forced to complete their training abroad. The law also means that invaluable laboratory engineers and senior technicians can no longer be retained under short-term contracts if a permanent position is not available. The nation urgently needs to put countermeasures in place, or risk losing crucial lab staff indefinitely.

Juan Iovanna INSERM UMR1068, CNRS UMR7258, Aix-Marseille University and Institute PaoliCalmettes, Marseille, France. juan.iovanna@inserm.fr

\section{Replace 'pathogens' with 'perceptogens'}

We argue for a more sweeping reappraisal of the term pathogen than Arturo Casadevall and Liise-anne Pirofski propose (Nature 516, 165-166; 2014). This should take in not just microbes, but the wider 'exposome' and recent discoveries in infection and immunity research.

A term is needed that encompasses sequences from the environment intrinsic or extrinsic - that impart pathogenic or benign information to eukaryotic immune receptors. For example, T-cell receptors that recognize autoantigen and microbial sequences can be triggered by related peptide sequences from diverse sources in the environment (M. E. Birnbaum et al. Cell 157, 1073-1087; 2014).
Substituting 'microbial immunogen' for 'pathogen' would not account for microbiota sequences that instruct immune development rather than elicit protective immunity. We suggest instead the term perceptogen (microbial or environmental) to cover protein sequences that affect the body's range of reactions after perception by its immune receptors.

As the writer Aldous Huxley remarked: "There are things known and there are things unknown and in between are the doors of perception."

Danny Altmann, Rosemary Boyton Imperial College London, UK.

d.altmann@imperial.ac.uk

\section{Learning from Typhoon Haiyan}

In our view, the communication of disaster risk during Typhoon Haiyan, which struck the Philippines in November 2013, could have been better.

The typhoon was one of the strongest tropical storms ever to make landfall, registering category 5 on the Saffir-Simpson scale. Despite forecasts of winds of more than 300 kilometres per hour and a predicted 7-metre storm surge, the city of Tacloban was caught underprepared: thousands died from the inundation.

The storm surge was predicted in a report by the Philippine Atmospheric, Geophysical and Astronomical Services Administration (PAGASA) that was sent to local agencies and communities. Unfortunately, it was simply a line at the end of a routine weather bulletin. It was apparently not otherwise highlighted, elaborated on or, in our opinion, in any way explained in order to transmit its urgency to key agencies and the public.

After interviewing agency personnel, we concluded that a well-intended adherence to routine and pro forma communication could have been at play. Feedback loops for conveying tacit information (for example, the implications of modelling outputs) seem to have been inadequate.

PAGASA's Tacloban team stayed in its single-storey coastal office, which was demolished by the storm surge, claiming a team member's life.

Many other factors influenced the impact of Haiyan, but this example indicates that routines need to adapt to deal with extreme events that lie beyond personal and institutional memory.

Raul Lejano New York

University, New York, USA. Joyce Melcar Tan, Meriwether Wilson University of Edinburgh, UK.

lejano@nyu.edu

\section{Build neuroscience capacity in Africa}

The non-profit organization TReND is funding a neuroscience training initiative so that Africa's scientists can join this rapidly evolving research field (see www.trendinafrica.org).

TReND (for 'Teaching and Research in Neuroscience for Development') is run by volunteer researchers at several universities worldwide. It organizes outreach courses and workshops for young African scientists on how to conduct quality, affordable neuroscience research in resource-limited settings. In 2010-14, more than 1,000 African students took part in TReND programmes.

The organization provides students with used lab equipment from universities, hospitals and companies in developed countries, as well as open-source software and hardware.

We call on industry and governments for more investment in such activities to improve science education and promote economic development in Africa. Fanuel Muindi, Joseph Keller Massachusetts Institute of Technology, Cambridge, USA. fmuindi@mit.edu 